\title{
Qualidade de vida de servidores técnico-administrativos: um estudo de caso
}

\author{
Valéria Cláudia Ferreira ANDRADE ${ }^{1}$ \\ Paulo César de Resende ANDRADE ${ }^{2}$ \\ Ângelo Márcio P. LEITE ${ }^{3}$ \\ Christiane. Motta ARAÚJO
}

\begin{abstract}
${ }^{1}$ Valéria Cláudia Ferreira ANDRADE. Aluna de Especialização/UFVJM - Campus II, Rodovia MGT 367, km 583, nº 5000, Alto da Jacuba, Diamantina, MG, 39100-000. E-mail: valeria.andrad@ hotmail.com

${ }^{2}$ Paulo César de Resende ANDRADE. Professor Dr. do Bacharelado em Ciência e Tecnologia/ICT/UFVJM - Campus II, Rodovia MGT 367, km 583, n 5000, Alto da Jacuba, Diamantina, MG, 39100-000. E-mail: paulo.andrade@ict.ufvjm.edu.br

${ }^{3}$ Ângelo Márcio P. LEITE. Professor Dr. da Engenharia Florestal/FCA/UFVJM - Campus II, Rodovia MGT 367, km 583, n 5000, Alto da Jacuba, Diamantina, MG, 39100-000. E-mail: ampleite@ig.com.br

${ }^{4}$ Christiane. Motta ARAÚJO. Professora da Enfermagem/FCBS/UFVJM - Campus II, Rodovia MGT 367, km 583, $\mathrm{n}^{\circ}$ 5000, Alto da Jacuba, Diamantina, MG, 39100-000. E-mail: christiane.motta@ufvjm.edu.br
\end{abstract}

\begin{abstract}
Resumo: A avaliação da qualidade de vida (QV) vem ganhando crescente valorização tanto no meio acadêmico, quanto na sociedade, e esse fenômeno vem sendo amplamente reconhecido nas últimas décadas. O objetivo deste trabalho foi descrever e avaliar os aspectos associados à qualidade de vida servidores técnico-administrativos da UFVJM, em Diamantina, buscando verificar a associação entre qualidade de vida e as variáveis demográficas relacionadas ao exercício da profissão, por intermédio da autopercepção destes. Foi estudada uma amostra de servidores em 2009 por meio da aplicação do questionário WHOQOL/Breve e calculado a média e o desvio-padrão para os escores deste questionário de QV. Utilizou-se o teste de Wilcoxon para comparar os gêneros. O escore médio do questionário de avaliação de QV foi 64,55, com maiores valores para o domínio físico e menores para meio ambiente. As diferenças das médias entre os gêneros não foram significativas $(\mathrm{p}>0,05)$ pelo teste de Wilcoxon, indicando não haver diferenças na QV entre homens e mulheres. Os profissionais apresentaram menor QV nos domínios meio ambiente e psicológico e maior nos domínios físico e relações sociais. Metade dos servidores considerou sua QV boa ou muito boa, uma vez estarem satisfeitos ou muito satisfeitos com relação a este aspecto.
\end{abstract}

Palavras-chave: qualidade de vida, funcionários públicos, autopercepção, fatores socioeconômicos, saúde ocupacional.

\begin{abstract}
The evaluating the quality of life (QL) is gaining increasing importance both in academia, society, and this phenomenon has been widely recognized in recent decades. The objective of this study was to describe and evaluate factors associated with quality of life of the administrative technician servers of the UFVJM, in Diamantina, trying to verify the association of quality of life with the demographic variables related to the profession, through the perception of these. The sample consisted of servers in 2009. The questionnaire WHOQOL/bref was applied and the mean and standard deviation was calculated for the scores of QL questionnaire. We used the Wilcoxon test to compare the genders. The average score of QL assessment questionnaire was 64.55, with higher values of the domain physical and smaller environment. The mean differences between genders were not significant $(\mathrm{p}>0.05)$ by Wilcoxon test, indicated no differences in QL between men and women. The professionals surveyed had lower QL in the areas environment and psychological and greater in physical and social relations. The half of the servers considered their QL as good or very good, once they are satisfied or very satisfied with this respect.
\end{abstract}

Keywords: quality of life, public employees, self-perception, socioeconomic factors, occupational health. 


\section{INTRODUÇÃO}

Qualidade de vida é a percepção do indivíduo tanto de sua posição na vida, no contexto da cultura e nos sistemas de valores nos quais se insere, como em relação aos seus objetivos, expectativas, padrões e preocupações (Spitzer, 1987). Segundo Bowling e Brasier (1995) é um conceito ligado ao desenvolvimento humano, implica estar em equilíbrio. Para garantir uma qualidade de vida satisfatória é necessário ter hábitos saudáveis, tempo para lazer, cuidar bem do corpo.

O conceito de QV é amplo uma vez poder ser afetado de modo complexo por diversos fatores entre os quais pela saúde física do indivíduo, pelo seu estado psicológico, por suas relações sociais, por seu nível de independência e pelas suas relações com as características mais relevantes do meio ambiente (Spitzer, 1987).

Para ter qualidade de vida o indivíduo deve manter outros hábitos que o façam se sentir bem, proporcionando conseqüências apropriadas como, usar o humor para lidar com situações de estresse, definir objetivos de vida e, o principal, sentir controle sobre a própria vida.

Os pesquisadores devem delinear claramente o que entende por QV e identificar os diversos domínios a serem avaliados, considerando que cada domínio identifica um foco particular de atenção e agrupa vários itens (Gill, 1994).
O WHOQOL (World Health Organization Quality of Life) é um questionário desenvolvido pela Organização Mundial de Saúde (OMS) para aferir a QV. Ele possui duas versões validadas para o português. O WHOQOL - 100 é composto por 100 questões e seis domínios: físico, psicológico, nível de independência, relações sociais, meio ambiente e aspectos religiosos. O WHOQOL/Breve é composto por 26 questões e quatro domínios: físico, psicológico, relações sociais e meio ambiente (Fleck et al, 2000).

A avaliação da QV tem despertado o interesse de pesquisadores, em diversas áreas do conhecimento, principalmente na saúde (Chaimowicz, 1997; Fleck et al, 2002; Nunes e Freire, 2006), sociologia e medicina (Bowlin e Brazier, 1995) e educação (Machado et al, 2011; Penteado e Pereira, 2007). O estudo da QV tem sido apontado como uma categoria analítica central para promover abordagens integradoras e interdisciplinares (Penteado e Pereira, 2007).

Machado et al (2011) fizeram um estudo sobre a qualidade de vida de docentes de cursos de graduação. A QV foi considerada boa ou muito boa.

O objetivo desta pesquisa foi descrever e avaliar os aspectos associados à QV dos servidores técnico-administrativos da Universidade Federal dos Vales do Jequitinhonha e Mucuri (UFVJM), na cidade de Diamantina; considerando os domínios, físico, psicológico, relações sociais e com o 
meio ambiente, buscando verificar a associação da QV com as variáveis demográficas relacionadas ao exercício da profissão, por intermédio da autopercepção destes.

\section{MATERIAIS E MÉTODOS}

A pesquisa foi realizada em maio de 2009 perante aos servidores técnicos administrativos da UFVJM, em Diamantina, Minas Gerais.

Os servidores técnicos administrativos foram selecionados por amostragem aleatória simples, tendo sido excluídos os que não estavam em atividade no período da coleta de dados, ou seja, inativos, de férias ou afastados por licenças médicas, maternidade, prêmio, interesse particular e à disposição de outros órgãos.

$\mathrm{Na}$ determinação do tamanho da amostra considerou-se um erro amostral máximo de $10 \%$ e intervalo de confiança de 95\%, com desvio-padrão aproximadamente de $1 / 4$ da diferença entre a amplitude das médias dos escores para os domínios. Com uma população de 60 servidores técnicos administrativos na data de realização da pesquisa, o tamanho amostral mínimo necessário foi de 14 pessoas, sendo realizada a coleta de dados (entrevistas) de 16 servidores.

A pesquisa foi realizada entre maio e agosto de 2009 perante aos servidores técnico-administrativos da UFVJM, em
Diamantina. A amostra participante da pesquisa foi significativa.

A escolha metodológica orientou-se no sentido de contemplar a subjetividade do funcionário como eixo primordial no levantamento dos dados; daí a opção por instrumentos disponíveis na literatura que permitissem aos sujeitos se auto-avaliar, a partir de suas percepções.

Foi utilizado como instrumento de coleta de dados o questionário WHOQOL/Breve. O WHOQOL/Breve contém 26 perguntas que envolvem aspectos diversos da vida cotidiana, das quais 24 são distribuídas em quatro domínios:

1) Físico: percepção do indivíduo sobre sua condição física. Contém as facetas: dor e desconforto; energia e fadiga; sono e repouso; atividades da vida cotidiana; dependência de medicação ou de tratamentos; capacidade de trabalho;

2) Psicológico: percepção do indivíduo sobre sua condição afetiva e cognitiva, cujas facetas são: sentimentos positivos; pensar, aprender, memória e concentração; auto-estima; imagem corporal e aparência; sentimentos negativos; espiritualidade/religião/ crenças pessoais;

3) Relações sociais: percepção do indivíduo sobre os relacionamentos sociais e os papéis sociais adotados na vida, com as seguintes facetas: relações pessoais; suporte (apoio) social; atividade sexual; e

4) Meio ambiente: percepção do indivíduo sobre aspectos diversos relacionados ao 
ambiente onde vive. Contém as facetas: segurança física e proteção; ambiente no lar; recursos financeiros; cuidados de saúde e sociais: disponibilidade e qualidade; oportunidades de adquirir novas informações e habilidades; participação em, e oportunidades de recreação/lazer; ambiente físico: (poluição / ruído / trânsito / clima); transporte.

Cada domínio é representado por várias facetas e suas questões foram formuladas com escala de intensidade (nada extremamente), capacidade (nada completamente), frequência (nunca - sempre) e avaliação (muito insatisfeito - muito satisfeito; muito ruim - muito bom).

O servidor pode apresentar sua resposta por meio de escores que variam de um a cinco (escala Likert), sendo a condição pior no escore um e a melhor, no escore cinco, para cada aspecto da qualidade de vida expresso no questionário WHOQOL/Breve.

Os resultados dos domínios apresentam valores entre zero e cem, sendo piores os mais próximos de zero e melhores, os mais próximos de cem. O cálculo dos domínios padronizados do WHOQOL/Breve segue a expressões definidas em função das perguntas constantes no questionário (WHOQOL, 1998).

Logan et al (1997) afirmam que o WHOQOL/Breve não prevê conceitualmente utilizar do escore global de qualidade de vida, então é calculado o escore de cada domínio, quanto mais alto o escore, melhor a qualidade de vida naquele domínio.

Além dos quatro domínios, o instrumento apresenta duas questões gerais, sendo que uma se refere à autopercepção da qualidade de vida e a outra sobre satisfação com a saúde.

Depois de coletados e tabulados, foi feita uma análise e o cálculo da média e do desvio-padrão dos escores padronizados dos domínios do protocolo WHOQOL/Breve. A comparação entre os gêneros foi feita pelo teste de Wilcoxon.

O instrumento de coleta de dados e o Termo de Consentimento Livre e Esclarecido do Sujeito - TCLE foram submetidos à apreciação e aprovação pelo Comitê de Ética em consonância com a Resolução 196/1996 do Conselho Nacional de Saúde, que estabelece normas éticas para pesquisas com seres humanos. Todos os participantes assinaram voluntariamente o TCLE, tendo sido informados de que a pesquisa não ofereceria nenhum tipo de risco dos pontos de vista físico e psicológico.

\section{RESULTADOS}

Foi feito um levantamento das características pessoais da população estudada, a fim de analisar alguns fatores e as suas contribuições para a QV, levando-se em conta o sexo, a faixa etária, estado civil, nível educacional, peso e altura. 
Com base nos dados da pesquisa constatou-se que há um equilíbrio dos servidores técnicos administrativos da UFVJM quanto ao gênero. O estado civil predominante é de solteiros $(66,7 \%)$.

A idade média é de 37,3 anos, faixa etária variando de 26 a 61 anos, com desviopadrão de 10 anos havendo, portanto, predominância de servidores jovens com idade abaixo dos 41 anos (62,5\%). Quanto ao nível de escolaridade pôde-se perceber que $37,5 \%$ dos servidores possui o $2^{\circ}$ grau completo e, com curso de pós-graduação completo, apenas $12,5 \%$.

O peso médio é de $71,5 \mathrm{Kg}$, variando de 53 a $92 \mathrm{~kg}$ e desvio-padrão 13,3 Kg, havendo, entretanto, equiparação de servidores com peso na faixa considerada ideal, entre 61 e $80 \mathrm{Kg}$, e fora desta faixa.

A altura média é de 1,69 m, variando de 1,48 a $1,88 \mathrm{~m}$ e desvio-padrão $0,12 \mathrm{~m}$ havendo, assim, $50 \%$ de servidores com altura entre 1,61 e $1,80 \mathrm{~m}$.

Determinou-se ainda o Índice de Massa Corporal dos servidores que relaciona a massa e a altura de um indivíduo. A maior parte dos homens $(66,7 \%)$ está com peso superior ao normal e, a maior parte das mulheres $(66,7 \%)$, estão com peso normal. Apesar dos homens normalmente apresentarem uma massa corpórea maior, eles apresentam também uma maior altura.

$\mathrm{O}$ escore médio referente a $\mathrm{QV}$ dos servidores técnicos administrativos da
UFVJM (WHOQOL/Breve) foi de 64,55 pontos.

$\mathrm{Na}$ Tabela 1 estão apresentados a média e o desvio-padrão para os escores padronizados dos domínios da QV.

Tabela 1 - Média e desvio-padrão dos escores dos domínios de qualidade de vida (WHOQOL/breve) de servidores da UFVJM, Diamantina, 2009.

\begin{tabular}{ccc}
\hline $\begin{array}{c}\text { Domínio da Qualidade de } \\
\text { Vida (WHOQOL/Breve) }\end{array}$ & Média & $\begin{array}{c}\text { Desvio- } \\
\text { padrão }\end{array}$ \\
\hline Físico & 71,7 & 13,0 \\
Psicológico & 66,4 & 12,8 \\
Relações Sociais & 67,2 & 17,1 \\
Meio Ambiente & 52,9 & 12,7
\end{tabular}

Fonte: Estudo da qualidade de vida de servidores da UFVJM, 2009.

A maior variação em termos de desvio-padrão ocorreu no domínio relações sociais e a menor, no domínio meio ambiente. O domínio meio ambiente mostrou-se o mais afetado, uma vez ter apresentado a menor média de escores, enquanto que o domínio físico com maior valor apresentou-se como o aspecto mais positivo da QV dos servidores da UFVJM.

As diferenças das médias entre os gêneros não foram significativas $(\mathrm{p}>0,05)$ pelo teste de Wilcoxon, indicando não haver diferenças na QV entre homens e mulheres, dentro do grupo de servidores estudados (Tabela 2). 
Tabela 2 - Média e desvio-padrão dos escores dos domínios de qualidade de vida (WHOQOL/breve), segundo o gênero dos servidores da UFVJM, Diamantina, 2009.

\begin{tabular}{ccc}
\hline $\begin{array}{c}\text { Domínio da Qualidade de } \\
\text { Vida (WHOQOL/Breve) }\end{array}$ & Feminino & Masculino \\
\hline Físico & $78,6^{\mathrm{ns}}$ & $67,9^{\mathrm{ns}}$ \\
Psicológico & $68,1^{\mathrm{ns}}$ & $61,8^{\mathrm{ns}}$ \\
Relações Sociais & $68,1^{\mathrm{ns}}$ & $68,1^{\mathrm{ns}}$ \\
Meio Ambiente & $57,8^{\mathrm{ns}}$ & $51,6^{\mathrm{ns}}$ \\
\hline
\end{tabular}

ns Não houve diferença significativa entre os gêneros ao nível de $5 \%$ de probabilidade.

Os dados sobre os aspectos que se destacaram, tanto negativa quanto positivamente, foram obtidos a partir da análise descritiva de cada uma das questões do WHOQOL/Breve.

Os resultados mostram que os aspectos que mais afetaram negativamente se relacionavam à vida profissional: baixa condição financeira, falta de informação e de oportunidade de lazer, além do desconforto no ambiente de trabalho, principalmente devido às condições de transporte e saúde.

Por outro lado, os aspectos que mais afetaram positivamente o grupo de servidores estudados relacionam-se à subjetividade, à vida pessoal e as questões físicas.

Quanto ao aspecto saúde, os servidores estão satisfeitos com sua condição, uma vez ter sido apontado por mais de oitenta por cento, pouca necessidade de algum tratamento médico para o desempenho de suas atividades. Este mesmo percentual afirmou ter plena capacidade para realizar o trabalho atual e, em torno de dois terços disseram estarem satisfeitos com sua capacidade de desempenhar as atividades diárias.

No tocante à aparência física verificou-se que a maior parte dos entrevistados $(60 \%)$, considera como muito boa esta dimensão e, portanto, aceita com tranquilidade a sua condição atual. Este resultado demonstra um bom grau de autoaceitação por parte dos servidores, constituindo um aspecto positivo e de fundamental importância para uma QV adequada.

Quanto aos sentimentos negativos, três quartos citaram que sentimentos como mau humor, desespero, ansiedade e depressão acontecem, mas com pouca frequência nas atividades diárias.

Em termos do grau de envolvimento nas relações pessoais verificou-se que mais da metade estão satisfeitos quanto a esta dimensão (amigos, parentes, conhecidos, colegas). O mesmo percentual disse estar satisfeitos ou bastante satisfeitos com a vida sexual, nível que pode ser considerado alto.

No que se refere ao domínio ambiente físico (clima, barulho, poluição, atrativos), menos da metade dos servidores disseram que o ambiente no qual estão inseridos é apenas medianamente saudável. Quanto à oportunidade de atividade de lazer, três quartos dos servidores classificaram este item como escasso. 
Em termos financeiros (remuneração recebida), a maioria dos servidores está pouco ou apenas mediamente satisfeita com o montante recebido mensalmente, dado as necessidades cotidianas (gastos). Quase a metade dos servidores está insatisfeita com o acesso a serviços de saúde e de transporte.

Quanto à auto-percepção do estado de saúde verificou-se que, a metade dos servidores técnicos administrativos considerou sua QV boa ou muito boa, uma vez estarem satisfeitos ou muito satisfeitos com relação a este aspecto.

Quando questionados sobre a condição atual de saúde, a maior parte a considerou boa ou muito boa, apesar de haver relatos de alguma condição insatisfatória ou problema de saúde.

Os principais problemas de saúde relatados foram hipertensão arterial, lesões osteoarticulares, doenças cardio vasculares e de hemorroidas. Outros problemas apontados em menor proporção foram: saúde mental, cansaço e dores lombares.

\section{DISCUSSÃO}

Esse é o primeiro estudo que utiliza o WHOQOL/Breve para avaliar a qualidade de vida de servidores técnico-administrativos de uma instituição de ensino superior. A discussão fica um pouco comprometida em função desse fato.

A auto-avaliação da QV, como era de se esperar, foi associada aos domínios, com exceção do domínio meio ambiente. Assim, quanto melhor a auto-percepção dos servidores sobre sua qualidade de vida, melhor sua qualidade de vida nos domínios físico, psicológico e relações sociais. Resultados semelhantes utilizando o WHOQOL foram encontrados nos trabalhos com acadêmicos da área de cirurgiõesdentistas (Nunes e Freire, 2006) e enfermagem (Saupe et al, 2004).

No Domínio Físico, a explicação para a maior parte dos servidores técnicos administrativos avaliados ter mencionado disposição (energia) e capacidade de desempenhar as atividades diárias se deve ao fato da maioria ter pouco tempo de exercício profissional na instituição e ainda estarem se adaptando ao trabalho, com disponibilidade de mostrar serviço com objetivos de crescimento individual concomitantemente com o crescimento da UFVJM. Esse resultado foi similar ao obtido por Machado et al (2011), quando da análise de docentes da mesma instituição.

A maior parte dos servidores disse estar satisfeitos com o seu estado de saúde e, apesar de incômodos ou alguma dor física manifestada de vez em quando, isto não dificulta ou os impede de realizar as atividades diárias, demonstrando pouca necessidade de intervenção (tratamento médico) e, portanto, indicador positivo para a QV.

No domínio Psicológico constatou-se que a maior parte dos entrevistados apresenta 
sentimentos negativos, mas com pouca frequência, o que não interfere na realização das atividades diárias. Outro aspecto positivo relacionado aos servidores refere-se à boa aceitação da aparência física atual (alto grau de auto-aceitação), constituindo também, aspecto de fundamental importância no maior grau de QV. O que mais se destaca é o fato de os servidores darem bastante sentido à vida, constituindo também, aspecto fundamental na qualidade de vida.

O domínio Relações Pessoais mostrou que grande parte dos entrevistados considera estar satisfeito com esta dimensão (amigos, parentes, conhecidos, colegas), estando também satisfeitos ou muito satisfeitos com o apoio que recebem destas pessoas. Resultado similar foi observado por Machado et al (2011). A explicação pode estar relacionada a uma vida mais saudável e tranquila dos servidores, em decorrência de Diamantina ser uma cidade de pequeno porte, que favorece as relações de amizade e o convívio familiar.

Quanto aos servidores estarem mediamente satisfeitos com relação ao domínio Ambiente, deve-se ao fato da instituição estar em construção / crescimento, não dispondo ainda de locais adequados de trabalho em termos de infraestrutura física e mobiliário. A oportunidade / disponibilidade de locais para atividades de lazer foi considerada de média a baixa. A insatisfação com a remuneração recebida foi considerada pelos servidores como média a baixa, tendo em vista o não atendimento por completo das necessidades cotidianas destes. Por sua vez, ressalta-se que isto não é um fato exclusivo da instituição, mas sim, um descontentamento geral de todos os servidores públicos federais do país. Verificou-se ainda que a qualidade dos serviços de saúde e de transporte, não foi considerada adequada. Machado et al (2011) afirmam que a qualidade de determinados serviços como saúde e transporte são responsáveis pelas piores pontuações no domínio.

\section{CONCLUSÃO}

A maioria dos entrevistados disse estar satisfeitos com sua saúde e, apesar de alguma dor física ou incômodo manifestado, isto não os impede de realizar as atividades diárias, mostrando pouca necessidade de intervenção (tratamento médico), o que caracteriza indicador positivo para a qualidade de vida.

Os servidores técnico-administrativos apresentaram menor QV nos domínios meio ambiente e relações social e maior nos domínios físico e psicológico.

\section{REFERÊNCIAS}

BOWLING, A. e BRAZIER, J. Quality of life in social science and medicine. Soc Sci ed. 1995. Vol. 41: 1337-1338.

CHAIMOWICZ, F. A saúde dos idosos brasileiros às vésperas do século XXI: problemas, projeções e alternativas. Rev. Saúde Pública. 1997. Vol. 31: 184-200. 
FLECK, M.P.A; LOUZADA, S.; XAVIER, M.; CHACHAMOVICH, E.; VIEIRA, G.; SANTOS, L.; PINZON, V. Aplicação da versão em português do instrumento abreviado de avaliação de qualidade de vida "WHOQOL/breve". Rev. Saúde Pública. 2000. Vol. 34: 178-183.

FLECK, M.P.A.; LIMA, A.F.B.S.; LOUZADA, S.; SCHESTATSKY, G.; HENRIQUES, A.; BORGES, V.X. Associação entre sintomas depressivos e funcionamento social em cuidados primários à saúde. Rev. Saúde Pública. 2002. Vol. 36: 431-438.

GILL, T.F. A critical appraisal of the quality of quality of life measurements. JAMA 1994. Vol. 272: 619-626.

LOGAN, H.L.; MULLER, P.J.; BERST, M.R.; YEANEY, D.W. Contributors to dentists job satisfaction and quality of life. J Am Coll Dent. 1997. Vol. 64(4): 39-43.

MACHADO, E. L.; ANDRADE, P.C.R; CAMPOS, C.A.; MACEDO, L.C.; ANDRADE, V.C.F. Qualidade de vida de docentes: um estudo de caso. Revista da Universidade Vale do Rio Verde, Três Corações, v. 9, n. 2, p. 255-263, ago./dez. 2011.

NUNES, M.F. e FREIRE, M.C.M. Qualidade de vida de cirurgiões-dentistas que atuam em um serviço público. Rev. Saúde Pública.

2006. Vol. 40 (6): 1019-1026.

PENTEADO, R.Z.; PEREIRA, I.M.T.B. Qualidade de vida e saúde vocal de professores. Rev. Saúde Pública. 2007. Vol. 41(2): 236-243.

SAUPE, R.; NIETCHE, E.A.; CESTAN, M.E.; GIORGI, M.D.M.; KRAHI, M. Qualidade de vida dos acadêmicos de enfermagem. Rev. Latinoam Enfermagem. 2004. Vol. 12: 636-642.

SPITZER, W.O. State of Science 1986:

Quality of life and functional status as target variables for research. J. Chronic Dis 1987. Vol. 40(6): 465-471.

WHOQOL Group. Development of the World Health Organization WHOQOL-Bref quality of life assessment. Psychol Med. 1998. Vol. 28: 551-558. 